\title{
COLOUR GRADIENTS IN CLUSTERS OF GALAXIES
}

\author{
M.D. SURAN AND N.A. POPESCU \\ Astronomical Institute of the Romanian Academy, \\ 75212 Bucharest 28, ROMANIA \\ e-mail:suran@roastro.astro.ro
}

In this paper we present a new indicator of the evolution of galaxies in clusters (both galactic and intrinsic cluster evolutionary phenomena) - the colour gradient in clusters of galaxies.

Our calculations were made for 16 clusters of galaxies and the results are presented in Table 1.

The individual values of the colour gradient (the slope $a=|\partial C / \partial R|$ of the C-R diagram for each cluster) present a cosmological dependence:

$$
\left|a_{\mathrm{B}-\mathrm{V}}\right|=0.522( \pm 0.071) z+0.264
$$

Our results are in good agreement with other evolutionary effects for clusters of galaxies. So, our indicator could be:

- directly related to Butcher-Oemler effect

$$
f_{\mathrm{B}}=0.533( \pm 0.15) z+0.043(n=14)
$$

- related to the environmental effect for different types of galaxies - the red galaxies prevail in the cores of clusters and blue galaxies in the outside regions;

- related to the new environmental effect for galaxies of the same type (Oemler et al. 1997, this Conference) - a high frequency of burst galaxies (blue and $\mathrm{E}+\mathrm{A}$ galaxies) outside the cluster density peak;

$\circ$ possibly related to the aperture, and with classes of compactness.

At the same time, the mean $\langle B-V\rangle$ colour per cluster indicates a direct connection with the galactic evolutionary process (on the "burst" track in the Rocca-Volmerange (1988) model for galactic evolution and an enhanced stellar activity between $0.4<z<0.6$ ).

These results seem to be in good agreement with the numerical simulations (Popescu \& Suran 1997, this volume), indicating an evolutionary 
time scale of $\sim 10$ Gyr. The obtained frequency of "E+A like" galaxies (post-starburst phenomena) in 6 clusters with $0.35<z<0.55$ is $\sim 3 \%$.

\section{References}

Rocca-Volmerange, B., \& Guiderdoni, B. 1988, A\&A.Suppl,74, 185

Popescu, N.A., \& Suran, M.D. 1996, Romanian Astron. J., 6 no.2, 105

TABLE 1. $f_{\mathrm{B}}, a_{\mathrm{B}-\mathrm{V}}$ and the mean colour in 16 clusters of galaxies

\begin{tabular}{|l|l|l|l|l|l|l|l|}
\hline Cluster & $z$ & $f_{\mathrm{B}}$ & $a_{\mathrm{B}-\mathrm{V}}^{\mathrm{E}}$ & $a_{\mathrm{B}-\mathrm{V}}^{\mathrm{S}}$ & $\langle B-V\rangle_{\text {roi }}$ & $\langle B-V\rangle^{\mathrm{E}}$ & $\langle B-V\rangle^{\mathrm{S}}$ \\
\hline Virgo & 0.0033 & 0.02 & -0.019 & -0.023 & 0.756 & 0.873 & 0.639 \\
Coma & 0.0235 & 0.011 & -0.076 & & 0.974 & 1.039 & 0.874 \\
A401 & 0.074 & 0.071 & -0.101 & -0.091 & 1.042 & 1.104 & 0.901 \\
3C 206 & 0.198 & 0.381 & -0.417 & & 1.443 & 1.607 & 0.87 \\
A1942 & 0.224 & 0.196 & -0.397 & -0.433 & 1.178 & 1.40 & 1.001 \\
$1455+2232$ & 0.259 & 0. & -0.409 & & 1.695 & 1.695 & 0.896 \\
$1358+6245$ & 0.32 & 0.152 & -0.49 & -0.514 & 1.469 & 1.709 & 1.159 \\
$0024+1654$ & 0.3909 & 0.182 & -0.416 & -0.447 & 1.868 & 2.102 & 1.337 \\
$0939+4713$ & 0.4069 & 0.321 & -0.38 & -0.448 & 1.797 & 2.036 & 1.33 \\
$0303+1706$ & 0.4181 & 0.4 & -0.495 & -0.486 & 1.743 & 2.059 & 1.184 \\
3C 295 & 0.46 & 0.263 & -0.524 & -0.624 & 1.677 & 2.071 & 1.22 \\
$1601+4253$ & 0.5391 & 0.301 & -0.548 & -0.463 & 1.715 & 2.109 & 1.194 \\
$0016+1609$ & 0.544 & 0.175 & -0.456 & -0.439 & 1.868 & 2.205 & 1.26 \\
$1305+2952$ & 0.62 & 0.476 & -0.579 & & 1.506 & 1.68 & 0.916 \\
3C 352 & 0.8 & 0.333 & -0.723 & & 1.284 & 1.405 & 0.75 \\
3C 265 & 0.81 & 0.625 & -0.726 & & 1.504 & 1.762 & 0.937 \\
\hline
\end{tabular}

